\title{
Exact Controllability and Stabilization for Kuramoto-Sivashinsky System
}

\author{
Carlos Frederico Vasconcellos ${ }^{1}$ \\ Instituto de Matemática e Estatística, UERJ, Rio de Janeiro, RJ \\ Patricia Nunes da Silva ${ }^{2}$ \\ Instituto de Matemática e Estatística, UERJ, Rio de Janeiro, RJ
}

\begin{abstract}
We analyze the stabilization and internal exact control for the KuramotoSivashinsky equation (KS) in a bounded interval. That is, we consider the following equation:

$$
u_{t}+\alpha u_{x x}+\beta u_{x x x}+\eta u_{x x x x}+u u_{x}=0 .
$$

The above equation is a model for small amplitude long waves. Using multiplier techniques we prove the exponential decay of the total energy associated with the KS system and in the linear case, using HUM method, we study its internal exact controllability.
\end{abstract}

Keywords. exponential decay; stabilization; Kuramoto-Sivashinsky equation

\section{Introduction}

We consider the Kuramoto-Sivashinsky system in a bounded interval $(0, L)$

$$
\mid \begin{aligned}
& u_{t}+\alpha u_{x x}+\beta u_{x x x}+\eta u_{x x x x}+u u_{x}=0 \quad \text { in } \quad(0, L) \times(0,+\infty) \\
& u(0, t)=u(L, t)=0 \quad \text { for all } \quad t \geq 0 \\
& u_{x}(0, t)=u_{x}(L, t)=0 \quad \text { for all } t \geq 0 \\
& u(x, 0)=u_{0} \quad \text { in } \quad(0, L) .
\end{aligned}
$$

Where $u_{0}$ belongs to $L^{2}(0, L)$ and the constant $\alpha$ is a negative real number, $\eta$ is positive and $\beta$ is non null.

The above system is a model for small amplitude long waves.

The equation:

$$
u_{t}+\alpha u_{x x}+\beta u_{x x x}+\eta u_{x x x x}+u u_{x}=0
$$

is known as the Kuramoto-Sivashinsky equation (KS) or as the generalized KuramotoSivashinsky equation. It is very important in describing physical processes in motion of

\footnotetext{
${ }^{1}$ cfredvasc@ime.uerj.br

${ }^{2}$ nunes@ime.uerj.br
} 
turbulence and other unstable process systems. The coefficients $\alpha, \beta$ and $\gamma$ are associated with instability, dispersion and dissipation effects, respectively. It is used to model reaction-diffusion systems (see for instance, Kuramoto [5] when $\beta=0$ ), flame-propagation and also viscous flow problems (see Sivashinsky [15] when $\beta=0$ ). Tadmor [16] showed the Cauchy problem connected to (1.2) when $\beta=0$ is well-posed, thus admitting a unique smooth solution, continuously dependent on its initial data. Uecker and Wierschem [17] studied the spatially periodic KS-equation (equation (1.2) with an aditional term $\delta(\cos (x) u)_{x}$ ) as a model for inclined film flow over wavy bottoms and other spatially periodic systems with a long wave instability. They also studied the stability and decay rate of the periodic solutions for such problem. The additional term $\delta(\cos (x) u)_{x}$ takes into account the flow of a viscous liquid film down an inclined plane, driven by gravity, when the bottom plate is not flat.

Larkin [6] also considered (1.2) in a bounded domain as a model of long waves in a viscous fluid flowing down on an inclined plane. In this paper, he proved that solutions to a mixed problem for the KdV equation may be obtained as singular limits of solutions to a corresponding mixed problem for the KS equation. He considered in (1.2), $\alpha=\eta$.

In [9], Iosevich and Miller refers to (1.2) as the Kuramoto-Sivashinsky/KdV equation. They were concerned with the case where the dispersive effects are important (that is, when the coefficient $\beta$ is large) and obtained solutions to the KdV equation as the limit of solutions of (1.2) with high dispersive and dissipative terms. To deal with a productiondissipation energy balance, Christov and Velarde [2] considered the Korteweg-de-VriesKuramoto-Sivashinsky-Velarde equation (it was added to equation (1.2) an extra nonlinear term $\left.\left(u u_{x}\right)_{x}\right)$. Is this way they were able to incorporate the Marangoni effect into the one-way long-wave assumption and to model a liquid layer being heated from above (from the air side).

This paper is devoted to study the decay of the energy, as $t \rightarrow+\infty$, associated to the system. (1.1)

In section 2, we begin by studying the linear case, that is when the term $u u_{x}$ is null. We prove global existence and uniqueness and some regularity for solutions. Then using multipliers techniques, we show the exponential decay of the energy associated to linear problem and we obtain some observability results. We also prove the internal exact controllability using the HUM method.

In section 3, we study the system (1.1). At first, we prove the existence and uniqueness of solutions and after we analyze the exponential decay of the energy associated with the system (1.1). This energy is defined by

$$
E(t)=\frac{1}{2} \int_{0}^{L}|u(x, t)|^{2} d x=\frac{1}{2}\|u(t)\|^{2} .
$$

Using the boundary conditions in (1.1) we prove that

$$
\frac{d E}{d t}=\alpha \int_{0}^{L}\left|u_{x}(x, t)\right|^{2} d x-\eta \int_{0}^{L}\left|u_{x x}(x, t)\right|^{2} d x \leq 0, \forall t>0 .
$$

We observe that, according to the above energy dissipation law the energy $E(t)$ is a nonincreasing function of the time. Then, we study the following question: 
Does the energy $E(t) \rightarrow 0$ as $t \rightarrow+\infty$ ? Is it possible to find a rate of decay of the energy? We show that the energy associated to problem (1.1) decays exponentially and this decay is uniform.

\section{The Linear System}

In this section we analyze the linear system associated with system (1.1).

In subsection 2.1, we begin by proving existence, uniqueness and regularity of solutions for the following system:

$$
\mid \begin{aligned}
& u_{t}+\alpha u_{x x}+\beta u_{x x x}+\eta u_{x x x x}=0 \quad \text { in } \quad(0, L) \times(0,+\infty) \\
& u(0, t)=u(L, t)=0 \quad \text { for all } \quad t \geq 0 \\
& u_{x}(0, t)=u_{x}(L, t)=0 \quad \text { for all } t \geq 0 \\
& u(x, 0)=u_{0} \quad \text { in } \quad(0, L) .
\end{aligned}
$$

Here $u_{0}$ belongs to $L^{2}(0, L)$ and the constant $\alpha$ is a negative real number, $\eta$ is positive and $\beta$ is non-null.

\subsection{Existence, uniqueness and regularity}

We shall use basically semigroups theory to prove the existence and uniqueness and to prove regularity of solutions we shall consider the multipliers techniques.

\section{Theorem 2.1. (Existence, uniqueness and regularity)}

If $u_{0}$ belongs to $L^{2}(0, L), \alpha<0, \eta>0$ and $\beta \neq 0$, then the problem (2.1) has a unique solution u belonging to $C\left([0,+\infty) ; L^{2}(0, L)\right) \cap L^{2}\left(0,+\infty ; H_{0}^{2}(0, L)\right)$. Moreover,

i) $\|u\|_{C\left([0,+\infty) ; L^{2}(0, L)\right)}+\|u\|_{L^{2}\left(0,+\infty ; H_{0}^{2}(0, L)\right)} \leq C\left\|u_{0}\right\|$.

ii) the energy dissipation law,

$$
\frac{d E}{d t}=\alpha \int_{0}^{L}\left|u_{x}(x, t)\right|^{2} d x-\eta \int_{0}^{L}\left|u_{x x}(x, t)\right|^{2} d x \leq 0, \quad \forall t>0 \text {, holds. }
$$

Sketch of Proof: Let $A v=-\alpha v^{\prime \prime}-\beta v^{\prime \prime \prime}-\eta v^{\prime \prime \prime \prime}$ denote the closed linear operator defined on the domain $D(A)=H^{4}(0, L) \cap H_{0}^{2}(0, L) \subset L^{2}(0, L)$.

So, using integration by parts and definition of $D(A)$, we can prove that:

$$
(v, A v)=\alpha \int_{0}^{L}\left|v^{\prime}\right|^{2} d x-\eta \int_{0}^{L}\left|v^{\prime \prime}\right|^{2} d x .
$$

On the other hand, we see that the adjoint operator $A^{*}$ is defined by $A^{*} w=-\alpha w^{\prime \prime}+\beta w^{\prime \prime \prime}-\eta w^{\prime \prime \prime \prime}$, where $w$ belongs to $D\left(A^{*}\right)=D(A)$.

So, using integration by parts again, we obtain:

$$
\left(w, A^{*} w\right)=\int_{0}^{L} w\left(-\alpha w^{\prime \prime}+\beta w^{\prime \prime \prime}-\eta w^{\prime \prime \prime \prime}\right) d x=\alpha \int_{0}^{L}\left|w^{\prime}\right|^{2} d x-\eta \int_{0}^{L}\left|w^{\prime}\right|^{2} d x .
$$


Now, since $\alpha<0$ and $\eta>0$, it follows by (2.2) and (2.3) that $A$ and $A^{*}$ are dissipative operators. Therefore, from classical results in semigroup theory (Pazy [11] cor.4.4, chapter 1 ), we know that $A$ generates a strongly continuous semigroup of contractions in $L^{2}(0, L)$. Let $\{S(t)\}_{t \geq 0}$ be the semigroup of contractions generated by $A$, then the mild solution $u(t)=S(t) u_{0}, t \geq 0$ belongs to $C\left([0,+\infty) ; L^{2}(0, L)\right)$ and

$$
\|u\|_{C\left([0,+\infty) ; L^{2}(0, L)\right)} \leq\left\|u_{0}\right\| .
$$

To show that $u \in L^{2}\left(0,+\infty ; H_{0}^{2}(0, L)\right)$ and the energy dissipation law, we first consider $u_{0} \in D(A)$, then we use multipliers techniques in $(2.1)$ and integrating by parts in $(0, L)$. Now, by boundary conditions and the density of $D(A)$ in $L^{2}(0, L)$, we prove the item i) and ii) and so we conclude the theorem.

\subsection{Exponential decay of the energy}

In this subsection we shall prove the decay of the energy associated to the system (2.1). As in [10], [18] and [19], we shall use multipliers techniques. The origin of this method can be found in Zuazua [20], see also Komornik [4].

In view of the energy dissipation law (Theorem 2.1, ii), in order to analyze the rate of decay of solutions, as $t \rightarrow+\infty$, it is natural to study the problem of observability.

Proposition 2.1. (A observability result)

Under the assumptions of Theorem 2.1 we have:

For all $L>0$, for all $T>0$ and for all $u_{0} \in L^{2}(0, L)$, there exists $C_{1}=C_{1}(L, T, \alpha, \eta)>0$ such that:

$$
\left\|u_{0}\right\|^{2} \leq C_{1}\left(-\alpha \int_{0}^{T} \int_{0}^{L}\left|u_{x}(x, t)\right|^{2} d x d t+\eta \int_{0}^{T} \int_{0}^{L}\left|u_{x x}(x, t)\right|^{2} d x d t\right) .
$$

it is suffice to prove the following inequality:

For any $T>0$, we have

$$
\frac{1}{2}\left\|u_{0}\right\|^{2} \leq \frac{1}{2 T} \int_{0}^{T} \int_{0}^{L}|u(x, t)|^{2} d x d t-\alpha \int_{0}^{T} \int_{0}^{L}\left|u_{x}(x, t)\right|^{2} d x d t+\eta \int_{0}^{T} \int_{0}^{L}\left|u_{x x}(x, t)\right|^{2} d x d t
$$

for all finite energy solutions of (2.1)

We consider $u_{0} \in D(A)$ and $T>0$, then we multiply the equation in $(2.1)$ by $(T-t) u$ and integrate on $(0, T) \times(0, L)$.

Again, we use integration by parts the boundary conditions and the density of $D(A)$ to conclude this proposition.

Now, we can to prove the main result of this subsection.

Theorem 2.2. (Exponential decay of the energy associated to linear problem) There exist $c>0$ and $\mu>0$ such that

$$
E(t) \leq c\left\|u_{0}\right\|^{2} e^{-\mu t}
$$

for all $t \geq 0$ and all solution of (2.1) with $u_{0} \in L^{2}(0, L)$. 
Proof: By (2.4) we can deduce that there exists a constant $C>0$ such that:

$$
E(0)=\frac{1}{2}\left\|u_{0}\right\|^{2} \leq C\left(-\frac{\alpha}{2} \int_{0}^{T} \int_{0}^{L}\left|u_{x}(x, t)\right|^{2} d x d t+\frac{\eta}{2} \int_{0}^{T} \int_{0}^{L}\left|u_{x x}(x, t)\right|^{2} d x d t\right) .
$$

So, using Theorem 2.1 and 2.5 we show that:

$$
E(T) \leq \frac{C}{1+C} E(0)
$$

So, by the semigroup property, the conclusion of Theorem 2.2 follows.

\subsection{Internal Exact Controllability}

In this subsection we analyze the internal exact controllability problem for the linear KS system.

We prove the following problem:

Given the initial and final states $\left(u_{0}, u_{T}\right)$ belonging to $L^{2}(0, L) \times L^{2}(0, L)$, it is possible to find a control function $h \in L^{2}\left(0, T ; H^{-2}(0, L)\right)$ such that the solution $v$ of the below system satisfies $v(x, T)=u_{T}(x), \forall x \in(0, L), \forall T>0$ and $\forall L \in(0,+\infty)$.

$$
\mid \begin{aligned}
& v_{t}+\alpha v_{x x}+\beta v_{x x x}+\eta v_{x x x x}=h \quad \text { in } \quad(0, L) \times(0, T) \\
& v(0, t)=v(L, t)=0 \quad \text { for all } \quad t \in(0, T) \\
& v_{x}(0, t)=v_{x}(L, t)=0 \quad \text { for all } t \in(0, T) \\
& v(x, 0)=u_{0} \quad \text { in } \quad(0, L) .
\end{aligned}
$$

where $\alpha<0, \eta>0$ and $\beta \neq 0$.

Before to answer this question, we need to show that the above system is well posed. In fact, it is possible to prove that:

Theorem 2.3. (Existence and uniqueness for system (2.6))

If $u_{0}$ belongs to $L^{2}(0, L), h$ belongs to $L^{2}\left(0, T ; H^{-2}(0, L)\right)$, with $\alpha<0, \eta>0$ and $\beta \neq 0$, then, for any $T>0$, the system (2.6) has a unique weak solution $v$ belonging to $X_{T}=C\left([0, T] ; L^{2}(0, L)\right) \cap L^{2}\left(0, T ; H_{0}^{2}(0, L)\right)$.

We will give a sketch of the proof of the global existence and uniqueness of solutions.

Consider $u_{0} \equiv 0$ and $h$ belonging to $L^{2}\left(0, T ; L^{2}(0, L)\right)$.

Let $\{S(t)\}_{t \geq 0}$ be the strongly continuous semigroup of contractions generated by the operator $A$ defined in the Theorem 2.1. Then applying the standard variation-of-parameters procedure, we have the mild solution of the problem (2.6).

So, using the density of $L^{2}\left(0, T ; L^{2}(0, L)\right)$ in $L^{2}\left(0, T ; H^{-2}(0, L)\right)$ and "a priori estimate' we conclude the theorem.

Theorem 2.4. (The internal exact controllability for system (2.6))

Let $L$ be in $(0,+\infty)$, then, for any $T>0$ and for any $\left(u_{0}, u_{T}\right) \in L^{2}(0, L) \times L^{2}(0, L)$, it exists $h \in L^{2}\left(0, T ; H^{-2}(0, L)\right)$ such that the solution $v$ of (2.6) satisfies $v(\cdot, T)=u_{T}$. 
To prove this Theorem we apply the HUM method (see, for instance Lions [8]) and multipliers techniques.

\section{$3 \quad$ The KS System(1.1)}

In this section we analyze the nonlinear system KS. We begin proving the existence and uniqueness of the solution and then we show the uniform exponential decay of the energy associated to the system (1.1).

Theorem 3.1. (Existence and uniqueness for system (1.1))

If $u_{0}$ belongs to $L^{2}(0, L), \alpha<0, \eta>0$ and $\beta \neq 0$ then, the problem (1.1) has a unique solution u belonging to $C\left([0,+\infty) ; L^{2}(0, L)\right) \cap L_{\text {loc }}^{2}\left(0,+\infty ; H_{0}^{2}(0, L)\right)$.

We prove the existence and uniqueness of the solution of the system (1.1)using semigroup theory, fixed point theorem and boundary conditions.

Theorem 3.2. (Exponential decay of the energy associated to problem (1.1)) There exist $c>0$ and $\mu>0$ such that

$$
E(t) \leq c\left\|u_{0}\right\|^{2} e^{-\mu t}
$$

for all $t \geq 0$ and all solution of (1.1) with $u_{0} \in L^{2}(0, L)$.

Since $\int_{0}^{T} \int_{0}^{L} u u_{x} u d x d t=0$, we can proceed like in the Theorem 2.2.

\section{Referências}

[1] T .B. Benjamin, J. L. Bona and J. J. Mahony, Model equations for long waves in nonlinear dispersive systems, Philos. Trans. Roy. Soc. London, Ser. A 272 (1972) $47-78$.

[2] C. I. Christov and M. G. Velarde, Dissipative solitons, Physica D: Nonlinear Phenomena, 86 (1995) 323-347.

[3] G. G. Doronin and N. A. Larkin, Kuramoto-Sivashinsky model for a dusty medium, Mathematical Methods in the Applied Sciences, 26 (2003) 179-19.

[4] V. Komornik, Exact controllability and Stabilization, the multiplier method, Research in Applied Mathematics, 36, John Willie and Sons, Masson, 1994.

[5] Y. Kuramoto, Instability and turbulence of wave fronts in reaction-diffusion systems, Progr. Theoret. Phys. 63 (1980) 1885-1903.

[6] N. A. Larkin, Korteweg-de Vries and Kuramoto-Sivashinsky equations in bounded domains, Journal of Mathematical Analysis and Applications, 297 (2004) 169-185. 
[7] F. Linares and A. F. Pazoto, On the exponential decay of the critical generalized Korteweg-de Vries with localized damping, Proc. Amer. Math. Soc. 135 (2007) 15151522 .

[8] J. L. Lions, Contrôlabilité Exacte, pertubations et stabilization de systèmes distribués, Tome 1. Contrôlabilité Exacte, Collection de Recherches en Mathématiques Appliquées, 8, Masson, Paris, 1988.

[9] A. Iosevich and J. Miller, Dispersive effects in a modified Kuramoto-Sivashinsky equation, Comm. PDE, 27 (2002) 2423-2448.

[10] G. P. Menzala, C.F.Vasconcellos and E.Zuazua, Stabilization of the Korteweg-de Vries equation with localized damping, Quarterly Applied Math. LX (2002) 111-129.

[11] A.Pazy, Semigroups of linear operators and applications to partial differential equations, Springer-Verlag, New York, 1983.

[12] L. Rosier and B. Y. Zhang, Global Stabilization of the Generalized Korteweg-De Vries Equation Posed on a Finite Domain, SIAM Journal on Control and Optimization 45 (2006) 927-956.

[13] P. N. da Silva and C. F. Vasconcellos, On the stabilization and controllability for a third order linear equation, Portugal.Math. 68 (2011) 279-296.

[14] P. N. da Silva and C. F. Vasconcellos, On the Kuramoto-Sivashinsky system in a bounded domain, FJMS, 71, 1(2012) 47-65

[15] G. Sivashinsky, Nonlinear analysis of hydrodynamic instability in laminar flames, Part I, Derivation of basic equations, Acta Astronaut. 4 (1977) 1117-1206.

[16] E. Tadmor, The well-posedness of the Kuramoto-Sivashinsky equation, SIAM J. Math. Anal. 17 (1986) 884-893.

[17] H. Uecker and A. Wierschem, A spatially periodic Kuramoto-Sivashinsky equation as a model problem for inclined film flow over wavy bottom, Electronic Journal of Differential Equations 2007118 (2007) 1-18.

[18] C. F. Vasconcellos and P. N. da Silva, Stabilization of the Linear Kawahara Equation with Localized Damping, Asymptotic Analysis 58 (2008) 229-252.

[19] C. F. Vasconcellos and P. N. da Silva, Stabilization of the Kawahara Equation with Localized Damping, ESAIM, Control. Optimization and Calculus of Variations 17 (2011) 102-116.

[20] E. Zuazua, Exponential decay for the semilinear wave equation with locally distribued damping, Comm. P.D.E. 15 (1990) 205-235. 